\title{
Newly discovered insect RNA viruses in China
}

\author{
QIU Yang*, WANG ZhaoWei, LIU YongXiang, QI Nan, SI Jie, XIANG Xue, \\ XIA XiaoLing, HU YuanYang ${ }^{*} \&$ ZHOU Xi
}

State Key Laboratory of Virology, College of Life Sciences, Wuhan University, Wuhan 430072, China

Received May 29, 2013; accepted June 17, 2013

\begin{abstract}
Insects are a group of arthropods and the largest group of animals on Earth, with over one million species described to date. Like other life forms, insects suffer from viruses that cause disease and death. Viruses that are pathogenic to beneficial insects cause dramatic economic losses on agriculture. In contrast, viruses that are pathogenic to insect pests can be exploited as attractive biological control agents. All of these factors have led to an explosion in the amount of research into insect viruses in recent years, generating impressive quantities of information on the molecular and cellular biology of these viruses. Due to the wide variety of insect viruses, a better understanding of these viruses will expand our overall knowledge of their virology. Here, we review studies of several newly discovered RNA insect viruses in China.
\end{abstract}

insect virus, Wuhan Nodavirus, Dendrolimus punctatus tetravirus, Ectropis obliqua picorna-like virus

Citation: Qiu Y, Wang Z W, Liu Y X, et al. Newly discovered insect RNA viruses in China. Sci China Life Sci, 2013, 56: 711-714, doi: 10.1007/s11427$013-4520-2$

From the view of biology, insects are the hosts of insect virus. However, a balanced relationship has been established between many insect viruses and their hosts. Research on insect viruses has two direct purposes: the protection of beneficial insects and biological control. Most importantly, due to the wide variety of insect viruses, studying these viruses and determining the similarities and differences among them will extend our understanding of virology and, moreover, facilitate the study of the important human viral pathogens. This review focuses on several newly discovered positive-strand RNA viruses isolated from insects in China, including nodavirus, a well-recognized model for studying RNA virus replication [1-7], tetravirus, a good model for studying the molecular driving forces for the evolution of positive-strand RNA viruses [8-10], and picorna-like virus, an ideal model for studying the molecular mechanism of important human pathogens as there is no concerns for its biological safety [11-17].

*Corresponding author (email: yyhu@whu.edu.cn; yangqiu@whu.edu.cn)

\section{Wuhan Nodavirus}

Wuhan Nodavirus (WhNV) is the first reported nodavirus isolated from insects in China [5]. As a member of the family Nodaviridae, $\mathrm{WhNV}$ is a non-enveloped virus with a $T=3$ icosahedral capsid. Its genome consists of two positivestrand RNAs: RNA1 (3.1 kb) and RNA2 (1.4 kb) [5,18]. RNA1 contains an open reading frame (ORF) for the synthesis of protein A, which serves as the RNA-dependent RNA polymerase (RdRp) for the amplification of both genomic strands, as well as a subgenomic RNA (sgRNA3). Protein A is the only virus-encoded protein required for genomic RNA replication and also suffices for the addition of cap-structures to the 5' ends of progeny RNA [19]. RNA2 encodes the precursor of the coat protein (protein $\alpha$ ), which is not infectious unless it undergoes autocatalytic cleavage [20]. WhNV sgRNA3 is $370 \mathrm{nt}$ in length, which is not packaged into virions, and encodes protein $\mathrm{B} 2$, a viral suppressor of RNA silencing in infected hosts [21-23].

WhNV sgRNA3 is synthesized via an internal initiation 
mechanism from its transcriptional start site (negativestrand RNA1, $2780 \mathrm{nt}$ ) [24]. The core promoter of WhNV sgRNA3 is between positions -22 to $+6 \mathrm{nt}$ of its transcriptional start site. The stem-loop structure of WhNV sgRNA3 is predicted upstream its transcriptional start site. Both the secondary structure and the primary sequence are required for promoter activity. Also, the synthesis of sgRNA3 is tightly coupled to the replication of RNA2 [24].

RNA interference (RNAi) is a eukaryotic gene silencing mechanism that functions as antiviral innate immunity in diverse organisms [25]. To combat RNAi immunity, viruses encode viral suppressors of RNA silencing (VSRs) that target RNA or protein components of the RNAi machinery [26]. WhNV B2 protein inhibits the host RNAi antiviral pathway at several levels [21]. For instance, WhNV B2 inhibits Dicer-mediated double-stranded (ds)RNA cleavage and the incorporation of siRNA into the RNA-induced silencing complex (RISC) by sequestering dsRNA and siRNA [22]. In addition, WhNV B2 protein suppresses the RNAi pathway by directly interacting with the PAZ and RNase III domains of Dicer-2 and blocking the activity of Dicer-2 via its C-terminal region [21]. Moreover, RNA binding enhances the B2-Dicer-2 interaction by promoting B2 homodimerization [21,22].

Despite being insect pathogens, nodaviruses have gained increasing attention in the scientific community as ideal model organisms for RNA virology and as vehicles for foreign protein presentation [27]. These nodavirus applications are mainly due to their following distinct features: (i) they share a simple genomic organization and replicate in a wide variety of cells [28,29]; (ii) they serve as valuable model systems to study the innate immunity of insects, particularly the induction and suppression of RNA silencing [30-37]; and (iii) they can be used as epitope presentation systems for the development of novel anthrax antitoxins and vaccines [38-41]. Due to these important features, a better understanding of the core steps of the nodaviral life cycle, such as replication and survival, is highly desired.

\section{Dendrolimus punctatus tetravirus}

Dendrolimus punctatus tetravirus (DpTV) was isolated from diseased Dendrolimus punctatus (pine moth) from Yunnan Province, China, and represents a new member of the genus Omegatetraviruses of the family Alphatetraviridae [42]. DpTV has a non-enveloped and icosahedral $T=4$ viral particle. The virus consists of two capsid proteins $(62.5$ and $6.8 \mathrm{kD})$ and two positive-strand RNAs (RNA1 and RNA2), which are 5.4 and $2.5 \mathrm{~kb}$ long, respectively [42].

RNA1 has a large ORF encoding a polypeptide of $~ 180$ $\mathrm{kD}$, while RNA2 contains two partially overlapping ORFs encoding polypeptides of 17 and $70 \mathrm{kD}$, respectively [42]. The $180 \mathrm{kD}$ protein contains consensus motifs of a putative methyltransferase, a helicase, and an RdRp, which shows significant similarity to those of other tetraviruses [42].

The replication of the positive-strand RNA viral genome consists of two steps: (i) synthesis of a complementary negative-strand RNA with the positive-strand genomic RNA as a template; and (ii) subsequent synthesis of progeny RNAs with the positive-strand RNA as a template. RdRps are central components in the life cycle of RNA viruses and are able to initiate RNA synthesis [43-45]. DpTV RdRp initiates viral RNA synthesis in a primer-independent manner but not via terminal nucleotidyl transferase activity in the presence of $\mathrm{Mg}^{2+}$ and an RNA template [46]. Mutation of the conversed RNA replication motif (GDD to GAA) abolishes the polymerase activity [46].

DpTV p17 protein is a RNA-binding protein [47]. When analyzed for its capacity to form oligomers, p17 was found to form dimers and tetramers. The p17 dimers bind to the RNA2 3'-UTR and RNA2 5'-UTR, as well as unrelated ssRNA and dsRNA, but not to the RNA1 3'-UTR. Thus, it appears that p17 dimers lack affinity for the RNA1 sequence. Further, the p17 tetramers only bind to the RNA2 3'-UTR, which indicates cooperative and specific properties of RNA binding. This specificity is both related to the cooperativity of p17 subunits in the tetramer and related to the sequence and structure of the RNA. The circular dichroism (CD) spectra of the viral RNA1 3'UTRs prove that their secondary structures are similar to yeast tRNAs [47]. The CD spectrum of the RNA1 3'-UTR indicates that its structure is slightly different from that of the RNA2 3'-UTR [47]. Perhaps these structural differences account for the varying affinities of the 17 protein to these UTRs.

Helicases normally utilize the energy generated from nucleoside triphosphate (NTP) hydrolysis to translocate along and unwind the helical structure of dsDNA or dsRNA $[48,49]$. Helicases are classified into six superfamilies (SF), SF-1 to SF-6 [49]. Sequence analysis predicts that the DpTV replicase contains a helicase domain (Hel). DpTV Hel is a functional RNA helicase, belonging to the SF-1 helicases, with 5'-3' dsRNA unwinding directionality [50]. The unwinding activity of DpTV Hel requires a 5' singlestranded tail on the RNA template and is dependent on reaction conditions. DpTV Hel contains NTPase activity, and the ATPase activity of DpTV Hel is significantly stimulated by dsRNA [50].

Tetraviruses have attracted much attention due to their close evolutionary linkage with nodaviruses, such as WhNV, that have $T=3$ capsids. The capsids of both tetraviruses and nodaviruses undergo autocatalytic cleavage before infection initiates [5,8-10,18]. Moreover, previous structural studies demonstrate numerous structural similarities between tetraviruses and nodaviruses, suggesting that tetraviruses are evolutionarily derived from nodaviruses, and betatetraviruses are evolutionarily more primordial and closer to nodaviruses than omegatetravirus [10]. Therefore, a better understanding of the biology of tetraviruses may both provide novel insights into the biological characteristics of the 
family Tetraviridae and help to elucidate the evolutionary relationship among betatetraviruses, omegatetraviruses, nodaviruses, and the alphavirus-like virus supergroup, as well as the molecular driving forces for the evolution of these positive-strand RNA viruses.

\section{Ectropis obliqua picorna-like virus}

Ectropis obliqua picorna-like virus (EoV) is a positivestrand RNA virus that leads to a lethal granulosis infection in the larvae of the tea looper (Ectropis obliqua) [51]. EoV was initially identified in Yunnan Province, China, and was classified as a member of the Iflaviridae family [51]. The family Iflaviridae comprises invertebrate viruses belonging to the picornavirus "superfamily" [52]. EoV possesses a large RNA genome $(9.4 \mathrm{~kb})$ encoding a single precursor polyprotein $(340 \mathrm{kD})$ that shares physicochemical properties with those of members of the family Picornaviridae, including an RdRp for viral RNA replication, a chymotrypsin-like $3 \mathrm{C}$ protease for proteolytic processing of picornaviral polyproteins into separate proteins, and a putative helicase (nonstructural protein 2C) [51-54].

The 5' UTR plays an important role in picornavirus translation initiation, as it contains an internal ribosome entry site (IRES) that mediates cap-independent translation [55]. The EoV IRES functions efficiently in both mammalian cell-derived systems and in an insect cell-derived translation system [56]. However, it functions inefficiently in a plant cell-derived translation system. Results show that deletions within the first $63 \mathrm{nt}$ have little impact on IRES activity, while core IRES function is contained within stem-loops $\mathrm{C}$ and D, as their removal significantly abrogates IRES activity [55]. Removal of stem-loop G, which contains two cryptic AUGs, causes a remarkable increase in IRES activity. The polypyrimidine tract (CCTTTC) has a slight effect on EoPV IRES activity.

The proteolytic processing of viral polyproteins is a crucial step in genome replication and capsid assembly in the order Picornavirales, which contains viruses whose genomes are generally translated a large precursor polyproteins. This maturation process is usually mediated by (more than one) proteases, and a $3 \mathrm{C}$ (for the family Picornaviridae) or 3C-like (3CL) protease (for other families), which plays a central role in the cleavage of the viral precursor polyprotein [57]. The EoV 3CL protease can release itself from neighboring domains via the localization of 3CL protease activity in the putative EoV 3CL region and the size of EoV 3CL protease [57]. The conserved H2261, D2299, and C2383 residues play critical roles in EoV 3CL protease activity. Moreover, N-terminal cleavage takes place in the E/A dipeptide at positions 2192 and 2193, and C-terminal cleavage occurs in the Q/S dipeptide at positions 2491 and 2492 [57].

Concerning the order Picornavirales, all of these viruses share several common features, such as virion structure and genomic organization. Thus, detailed molecular studies of EoV may provide novel insights into the biological features of various viruses in the order Picornavirales and, possibly, help uncover evolutionary driving forces in this group of positive-strand RNA viruses.

\section{Conclusion and perspectives}

Unlike human viruses, the study of insect viruses is not central to virology because human viruses are directly involved in human health. However, as the largest group of biological resources on Earth, insect viruses can be used as ideal models for studying the molecular details of viruses, the expression of exogenous proteins, and the biological control of insect pests. Therefore, their significance both in basic scientific research and practical applications should not be neglected.

We thank Ms. Markeda Wade for professionally editing this manuscript. This work was supported by the National Natural Science Foundation of China (31270190 and 81201292 to Zhou Xi, 31270189 to Hu YuanYang) and the Chinese 111 Project (B06018).

1 Miller D J, Schwartz M D, Ahlquist P. Flock house virus RNA replicates on outer mitochondrial membranes in Drosophila cells. J Virol, 2001, 75: 11664-11676

2 Eckerle L D, Ball L A. Replication of the RNA segments of a bipartite viral genome is coordinated by a transactivating subgenomic RNA. Virology, 2002, 296: 165-176

3 Miller D J, Ahlquist P. Flock house virus RNA polymerase is a transmembrane protein with amino-terminal sequences sufficient for mitochondrial localization and membrane insertion. J Virol, 2002, 76: 9856-9867

4 Lu R, Maduro M, Li F, et al. Animal virus replication and RNAimediated antiviral silencing in Caenorhabditis elegans. Nature, 2005, 436: 1040-1043

5 Liu C, Zhang J, Yi F, et al. Isolation and RNA1 nucleotide sequence determination of a new insect nodavirus from Pieris rapae larvae in Wuhan city, China. Virus Res, 2006, 120: 28-35

6 van Wynsberghe P M, Chen H R, Ahlquist P. Nodavirus RNA replication protein a induces membrane association of genomic RNA. J Virol, 2007, 81: 4633-4644

7 Stapleford K A, Rapaport D, Miller D J. Mitochondrion-enriched anionic phospholipids facilitate flock house virus RNA polymerase membrane association. J Virol, 2009, 83: 4498-4507

8 Bothner B, Taylor D, Jun B, et al. Maturation of a tetravirus capsid alters the dynamic properties and creates a metastable complex. Virology, 2005, 334: 17-27

9 Tomasicchio M, Venter $\mathrm{P}$ A, Gordon $\mathrm{K} \mathrm{H}$, et al. Induction of apoptosis in Saccharomyces cerevisiae results in the spontaneous maturation of tetravirus procapsids in vivo. J Gen Virol, 2007, 88: 1576-1582

10 Speir J A, Taylor D J, Natarajan P, et al. Evolution in action: N and C termini of subunits in related $T=4$ viruses exchange roles as molecular switches. Structure, 2010, 18: 700-709

11 Koonin E V, Wolf Y I, Nagasaki K, et al. The Big Bang of picornalike virus evolution antedates the radiation of eukaryotic supergroups. Nat Rev Microbiol, 2008, 6: 925-939

12 Wolthers K C, Benschop K S, Schinkel J, et al. Human parechoviruses as an important viral cause of sepsislike illness and meningitis in young children. Clin Infect Dis, 2008, 47: 358-363

13 de Almeida M B, Zerbinati R M, Tateno A F, et al. Rhinovirus C and 
respiratory exacerbations in children with cystic fibrosis. Emerg Infect Dis, 2010, 16: 996-999

14 Liu Y, Wang C, Mueller S, et al. Direct interaction between two viral proteins, the nonstructural protein $2 \mathrm{C}$ and the capsid protein VP3, is required for enterovirus morphogenesis. PLoS Pathog, 2010, 6: e1001066

15 Papageorgiou N, Coutard B, Lantez V, et al. The 2C putative helicase of echovirus 30 adopts a hexameric ring-shaped structure. Acta Crystallogr D Biol Crystallogr, 2010, 66: 1116-1120

16 Norder H, De Palma A M, Selisko B, et al. Picornavirus nonstructural proteins as targets for new anti-virals with broad activity. Antiviral Res, 2011, 89: 204-218

17 Gladue D P, O'Donnell V, Baker-Branstetter R, et al. Foot-andmouth disease virus nonstructural protein $2 \mathrm{C}$ interacts with Beclin1, modulating virus replication. J Virol, 2012, 86: 12080-12090

18 Liu C, Zhang J, Wang J, et al. Sequence analysis of coat protein gene of Wuhan nodavirus isolated from insect. Virus Res, 2006, 121: 17-22

19 Gallagher T M, Friesen P D, Rueckert R R. Autonomous replication and expression of RNA 1 from black beetle virus. J Virol, 1983, 46: $481-489$

20 Schneemann A, Zhong W, Gallagher T M, et al. Maturation cleavage required for infectivity of a nodavirus. J Virol, 1992, 66: 6728-6734

21 Qi N, Zhang L, Qiu Y, et al. Targeting of dicer-2 and RNA by a viral RNA silencing suppressor in Drosophila cells. J Virol, 2012, 86: 5763-5773

22 Qi N, Cai D, Qiu Y, et al. RNA binding by a novel helical fold of b2 protein from Wuhan Nodavirus mediates the suppression of RNA interference and promotes B2 dimerization. J Virol, 2011, 85: 9543-9554

23 Cai D, Qiu Y, Qi N, et al. Characterization of Wuhan Nodavirus subgenomic RNA3 and the RNAi inhibition property of its encoded protein B2. Virus Res, 2010, 151: 153-161

24 Qiu Y, Cai D, Qi N, et al. Internal initiation is responsible for synthesis of Wuhan Nodavirus subgenomic RNA. J Virol, 2011, 85: $4440-4451$

25 Ding S W, Voinnet O. Antiviral immunity directed by small RNAs. Cell, 2007, 130: 413-426

26 Baulcombe D. RNA silencing in plants. Nature, 2004, 431: 356-363

27 Venter P A, Schneemann A. Recent insights into the biology and biomedical applications of Flock House virus. Cell Mol Life Sci, 2008, 65: 2675-2687

28 Price B D, Rueckert R R, Ahlquist P. Complete replication of an animal virus and maintenance of expression vectors derived from it in Saccharomyces cerevisiae. Proc Natl Acad Sci USA, 1996, 93: 9465-9470

29 Price B D, Eckerle L D, Ball L A, et al. Nodamura virus RNA replication in Saccharomyces cerevisiae: heterologous gene expression allows replication-dependent colony formation. J Virol, 2005, 79: 495-502

$30 \mathrm{Li} \mathrm{H}, \mathrm{Li} \mathrm{W} \mathrm{X}$, Ding $\mathrm{S} \mathrm{W}$. Induction and suppression of RNA silencing by an animal virus. Science, 2002, 296: 1319-1321

31 Chao J A, Lee J H, Chapados B R, et al. Dual modes of RNAsilencing suppression by Flock House virus protein B2. Nat Struct Mol Biol, 2005, 12: 952-957

32 Lingel A, Simon B, Izaurralde E, et al. The structure of the flock house virus B2 protein, a viral suppressor of RNA interference, shows a novel mode of double-stranded RNA recognition. EMBO Rep, 2005, 6: 1149-1155

33 Wang X H, Aliyari R, Li W X, et al. RNA interference directs innate immunity against viruses in adult Drosophila. Science, 2006, 312: $452-454$

34 Ishikawa T, Matsumoto A, Kato T Jr., et al. DCRY is a Drosophila photoreceptor protein implicated in light entrainment of circadian rhythm. Genes Cells, 1999, 4: 57-65

35 Sullivan C S, Ganem D. A virus-encoded inhibitor that blocks RNA interference in mammalian cells. J Virol, 2005, 79: 7371-7379

36 Fenner B J, Thiagarajan R, Chua H K, et al. Betanodavirus B2 is an RNA interference antagonist that facilitates intracellular viral RNA accumulation. J Virol, 2006, 80: 85-94

37 Johnson K L, Price B D, Eckerle L D, et al. Nodamura virus nonstructural protein B2 can enhance viral RNA accumulation in both mammalian and insect cells. J Virol, 2004, 78: 6698-6704

38 Manayani D J, Thomas D, Dryden K A, et al. A viral nanoparticle with dual function as an anthrax antitoxin and vaccine. PLoS Pathog, 2007, 3: 1422-1431

39 Chen Y, Xiong X, Liu X, et al. Immunoreactivity of HCV/HBV epitopes displayed in an epitope-presenting system. Mol Immunol, 2006, 43: 436-442

40 Peng M, Dai C B, Chen Y D. Expression and immunoreactivity of an epitope of $\mathrm{HCV}$ in a foreign epitope presenting system. World $\mathrm{J}$ Gastroenterol, 2005, 11: 3363-3367

41 Venter P A, Schneemann A. Assembly of two independent populations of flock house virus particles with distinct RNA packaging characteristics in the same cell. J Virol, 2007, 81: 613-619

42 Yi F, Zhang J, Yu H, et al. Isolation and identification of a new tetravirus from Dendrolimus punctatus larvae collected from Yunnan Province, China. J Gen Virol, 2005, 86: 789-796

43 Hansen J L, Long A M, Schultz S C. Structure of the RNA-dependent RNA polymerase of poliovirus. Structure, 1997, 5: 1109-1122

44 Ahlquist P, Noueiry A O, Lee W M, et al. Host factors in positivestrand RNA virus genome replication. J Virol, 2003, 77: 8181-8186

45 Tao Y J, Ye Q. RNA virus replication complexes. PLoS Pathog, 2010, 6: e1000943

46 Zhou L, Zhang J, Wang X, et al. Expression and characterization of RNA-dependent RNA polymerase of Dendrolimus punctatus tetravirus. J Biochem Mol Biol, 2006, 39: 571-577

47 Zhou L, Zheng $\mathrm{Y}$, Jiang $\mathrm{H}$, et al. RNA-binding properties of Dendrolimus punctatus tetravirus p17 protein. Virus Res, 2008, 138: 1-6

48 Mackintosh S G, Raney K D. DNA unwinding and protein displacement by superfamily 1 and superfamily 2 helicases. Nucleic Acids Res, 2006, 34: 4154-4159

49 Singleton M R, Dillingham M S, Wigley D B. Structure and mechanism of helicases and nucleic acid translocases. Annu Rev Biochem, 2007, 76: 23-50

50 Wang Q, Han Y, Qiu Y, et al. Identification and characterization of RNA duplex unwinding and ATPase activities of an alphatetravirus superfamily 1 helicase. Virology, 2012, 433: 440-448

51 Wang X, Zhang J, Lu J, et al. Sequence analysis and genomic organization of a new insect picorna-like virus, Ectropis obliqua picorna-like virus, isolated from Ectropis obliqua. J Gen Virol, 2004, 85: $1145-1151$

52 Le Gall O, Christian P, Fauquet C M, et al. Picornavirales, a proposed order of positive-sense single-stranded RNA viruses with a pseudo-T=3 virion architecture. Arch Virol, 2008, 153: 715-727

53 van Oers M M. Genomics and biology of Iflaviruses. In: Asgari S, Johnson K, eds. Insect Virology. Norflok: Caister Academic Press, 2010. 231-250

54 Banerjee R, Weidman M K, Echeverri A, et al. Regulation of poliovirus $3 \mathrm{C}$ protease by the $2 \mathrm{C}$ polypeptide. J Virol, 2004, 78: 9243-9256

$55 \mathrm{Lu} \mathrm{J}$, Zhang J, Wang X, et al. In vitro and in vivo identification of structural and sequence elements in the $5^{\prime}$ untranslated region of Ectropis obliqua picorna-like virus required for internal initiation. J Gen Virol, 2006, 87: 3667-3677

$56 \mathrm{Lu} \mathrm{J}, \mathrm{Hu} \mathrm{Y}, \mathrm{Hu} \mathrm{L}$, et al. Ectropis obliqua picorna-like virus IRES-driven internal initiation of translation in cell systems derived from different origins. J Gen Virol, 2007, 88: 2834-2838

57 Ye S, Xia H, Dong C, et al. Identification and characterization of Iflavirus 3C-like protease processing activities. Virology, 2012, 428: $136-145$

Open Access This article is distributed under the terms of the Creative Commons Attribution License which permits any use, distribution, and reproduction in any medium, provided the original author(s) and source are credited. 